\title{
Association between socio-economic status and childhood undernutrition in Bangladesh; a comparison of possession score and poverty index
}

\author{
Masuda Mohsena*, CG Nicholas Mascie-Taylor and Rie Goto \\ Department of Biological Anthropology, University of Cambridge, Pembroke Street, Cambridge CB2 3RA, UK
}

Submitted 14 December 2009: Accepted 10 May 2010: First published online 25 June 2010

\begin{abstract}
Objective: To determine how much of the variation in nutritional status of Bangladeshi children under 5 years old can be attributed to the socio-economic status of the family.

Design: Nutritional status used reference $Z$-scores of weight-for-age (WAZ), height-for-age (HAZ) and weight-for-height (WHZ). A 'possession score' was generated based on ownership of a radio, television, bicycle, motorcycle and telephone, and the availability of electricity, with categories of 0 to $4+$ possessions. A five-point (quintile) 'poverty index' was created using principal component analysis.

Setting: The Bangladesh Demographic and Health Survey 2004 was the source of data. Subjects: A sample of 4891 children aged $<5$ years was obtained.

Results: Some $57 \cdot 8 \%$ of the sample was either stunted, wasted or underweight $(7 \cdot 7 \%$ were stunted, wasted and underweight). Of those stunted (48.4\%), 25.7\% were also underweight. Underweight and wasting prevalences were $40 \cdot 7 \%$ and $14 \cdot 3 \%$, respectively. Mean WAZ, HAZ and WHZ did not differ by sex. Children of mothers with no education or no possessions were, on average, about 1 SD more underweight and stunted than those with higher educated mothers or with $4+$ possessions. The possession score provided much greater discrimination of undernutrition than the poverty index. Nearly $50 \%$ of children from households with no possessions were stunted, wasted or underweight (only $27 \%$ in the poorest quintile), compared with only $3-6 \%$ of children from households with $4+$ possessions (over $13 \%$ in the richest quintile).

Conclusions: Maternal education and possession score were the main predictors of a child's nutritional status. Possession score was a much better indicator of undernutrition than the poverty index.
\end{abstract}

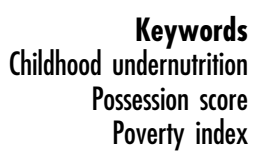

Childhood malnutrition is currently the leading cause of the global burden of disease in low- and middle-income countries. Recent estimates published in the Lancet $^{(1)}$ suggest that malnutrition is the underlying cause of 3.5 million deaths and $35 \%$ of the disease burden in children under 5 years of age. The fraction of total global health loss attributable to undernutrition was $9.5 \%$ in 2000 and $14.9 \%$ in high-mortality developing regions ${ }^{(2)}$. Ending malnutrition is an agenda of the current millennium, particularly for the developing world ${ }^{(3)}$. Lowering mortality and malnutrition rates among children, along with reducing the gender disparities in both these measures, have been identified as key Millennium Development Goals (MDG) for Bangladesh ${ }^{(4)}$.

Socio-economic status (SES) has long been identified as a leading predictive variable of an individual's health in the epidemiological, economic and sociological literature $^{(5,6)}$. However, international agencies have recently downplayed the importance of SES as a marker of malnutrition. For example, Helen Keller International ${ }^{(7)}$ reported that the highest and lowest SES quintile in Bangladesh differed only by $15 \%$ in the prevalence of stunting and Semba et $a l .{ }^{(8)}$ reported only $17 \cdot 9 \%$ higher prevalence of stunting among the poorest quintile compared with richest quintile. These findings suggest less importance of using SES to derive health policy recommendations.

It is well acknowledged that there is no international consensus on assessing SES. Income and household expenditures are the most commonly used measures of $\mathrm{SES}^{(9)}$. Given the difficulties in obtaining income or expenditure in developing countries, a poverty index or 
possession score has long been used as an alternative in measuring $\operatorname{SES}^{(5,10)}$.

In a developing country like Bangladesh, where effective allocation of limited resources is very crucial especially at the beginning of recent economic recession, comparative analysis of different socio-economic indicators for understanding the nature and determinants of malnutrition is essential. The aim of the present study was therefore to assess the magnitude of inequalities in malnutrition of children under 5 years old that are ascribable to SES and to see whether a possession score or poverty index best explains the extent of undernutrition in Bangladesh.

\section{Methodology}

The Bangladesh Demographic and Health Survey 2004 was the source of data, which contained information on 11400 women. The present study focused on the nutritional status of children under 5 years old. In total, 5125 families had at least one child below 5 years old, 1082 had two children, 130 families had three children and eight families had four children aged $<5$ years. In the analysis presented here, data on 4093 children, whose records were complete in the required individual and household level variables, were included; of whom 798 families had a second child.

Anthropometric indices, namely $Z$-scores of weight-forage (WAZ), height-for-age (HAZ) and weight-for-height (WHZ), were generated by using WHO 2006 reference values. The $Z$-scores of the children were analysed as continuous as well as categorical variables. The usual $Z$-score cut-off of -3 SD was used for classifying a child as severely malnourished and $Z$-score cut-off of -2 SD for classifying as malnourished. The socio-economic and demographic information included in the study were family size, parent's education and occupation, region (six divisions), residence (urban/rural), housing condition (type of floor, wall and roof), ownership of radio, television, bicycle, motorcycle and telephone, availability of electricity, type of cooking fuel, water supply and latrine, and family type. A new variable called 'possession score' was created using information about ownership of a radio, television, bicycle, motorcycle and telephone and the availability of electricity. All of these variables were binary, having 'yes' (1) or 'no' (0) categories, and adding these gave scores ranging from 0 to 6 . The possession variable was then categorised into five categories of 0 to $4+$, where 0 means the household had no assets and $4+$ means that family had four or more assets. One- or twochild families were defined by the variable family type. For relative comparison with possession score, a 'poverty index' was created using the technique of principal component analysis (PCA); the first component was taken and the scores on that component were divided into quintiles to reflect the poorest to wealthiest households.
All continuous data were checked for skewness using the Cox test (coefficient of skewness divided by standard error of skewness) as well as by examination of the frequency distribution with a normal curve. The relationship between two categorical variables was analysed by the $\chi^{2}$ test and between continuous variables by the independent-sample $t$ test. One-way ANOVA using either the Hochberg or Games-Howell post hoc test was used in comparing the means of three or more groups. Curve estimation was done to include the quadratic effects of the continuous independent variables. General linear models (GLM) were used to analyse dependent continuous variables with two or more independent variables. When the $Z$-scores were analysed as categorical variables, binary logistic regression analyses were undertaken with the socio-economic and demographic variables. Sequential models were mainly used in the analyses. For each dependent variable two types of analysis were carried out. In the first set (analysis 1), adjustment was made for linear and quadratic effects of age and family type, before testing for the effect of any variable separately. In the second analysis (analysis 2), all the other explanatory variables in the model, as well as linear and quadratic terms of age and family type, were entered in the analysis before testing the variable of interest. In every regression model, the variance inflation factor, tolerance and Cook's distance were calculated to check for multicollinearity and outliers. Because two children were used from the same family, all analyses were weighted and heterogeneity between one- and two-child families was also taken into account. Level of significance was taken at $P<0 \cdot 05$. Bonferroni corrections, both strict and sequential, were applied to correct for the number of statistical tests undertaken.

\section{Results}

The analysis (Table 1) revealed that $57 \cdot 8 \%$ of sample was suffering from some form of undernutrition (i.e. stunted, wasted or underweight); of those stunted (48.4\%), $25 \cdot 7 \%$ were also underweight while a further $7 \cdot 7 \%$ were stunted, wasted and underweight. Most of the Bangladeshi children were suffering from chronic malnutrition (i.e. stunting) or chronic/acute mixed malnutrition (i.e. underweight) and

Table 1 Breakdown of sample by stunting, underweight and wasting categories: children aged $<5$ years, Bangladesh Demographic and Health Survey 2004

\begin{tabular}{lrr}
\hline Category & $n$ & $\%$ \\
\hline Normal & 2063 & $42 \cdot 2$ \\
Stunted only & 732 & $15 \cdot 0$ \\
Underweight only & 137 & $2 \cdot 8$ \\
Wasted only & 104 & $2 \cdot 1$ \\
Stunted and underweight & 1258 & $25 \cdot 7$ \\
Underweight and wasted & 220 & $4 \cdot 5$ \\
Stunted, underweight and wasted & 377 & $7 \cdot 7$ \\
\hline
\end{tabular}


Table 2 Relationship between Z-scores and socio-economic and demographic variables: children aged $<5$ years, Bangladesh Demographic and Health Survey 2004

\begin{tabular}{|c|c|c|c|c|c|c|c|c|c|c|}
\hline & & & \multicolumn{4}{|c|}{ Analysis 1} & \multicolumn{4}{|c|}{ Analysis 2} \\
\hline & & & Mean & Difference & $F$ & $P$ & Mean & Difference & $F$ & $P$ \\
\hline \multirow[t]{12}{*}{ WAZ } & Mother's education level & No education & $-1 \cdot 98$ & 0.97 & $98 \cdot 72$ & $<0.001$ & $-1 \cdot 76$ & 0.43 & $9 \cdot 39$ & $<0.001$ \\
\hline & & Primary & $-1 \cdot 86$ & $0 \cdot 85$ & & & $-1 \cdot 72$ & $0 \cdot 39$ & & \\
\hline & & Secondary & $-1 \cdot 58$ & 0.57 & & & $-1 \cdot 60$ & $0 \cdot 27$ & & \\
\hline & & Higher & $-1 \cdot 01$ & 0 & & & $-1 \cdot 33$ & 0 & & \\
\hline & Possession score & 0 & $-2 \cdot 00$ & $0 \cdot 88$ & $81 \cdot 79$ & $<0.001$ & $-1 \cdot 80$ & 0.51 & $15 \cdot 33$ & $<0.001$ \\
\hline & & 1 & $-1 \cdot 88$ & $0 \cdot 76$ & & & $-1 \cdot 76$ & 0.46 & & \\
\hline & & 2 & $-1 \cdot 66$ & 0.55 & & & $-1 \cdot 64$ & $0 \cdot 35$ & & \\
\hline & & 3 & $-1 \cdot 46$ & $0 \cdot 34$ & & & $-1 \cdot 51$ & $0 \cdot 21$ & & \\
\hline & & $4+$ & $-1 \cdot 12$ & 0 & & & $-1 \cdot 30$ & 0 & & \\
\hline & Toilet & No toilet & $-2 \cdot 09$ & 0.62 & $94 \cdot 27$ & $<0.001$ & $-1 \cdot 71$ & $0 \cdot 21$ & $5 \cdot 79$ & 0.003 \\
\hline & & Insanitary & $-1 \cdot 87$ & $0 \cdot 40$ & & & $-1 \cdot 60$ & 0.09 & & \\
\hline & & Sanitary & $-1 \cdot 47$ & 0 & & & $-1 \cdot 50$ & 0 & & \\
\hline \multirow[t]{20}{*}{ HAZ } & Region & Barisal & $-2 \cdot 19$ & $0 \cdot 15$ & $12 \cdot 28$ & $<0.001$ & $-1 \cdot 96$ & $0 \cdot 17$ & $7 \cdot 83$ & $<0.001$ \\
\hline & & Chittagong & $-2 \cdot 06$ & 0.02 & & & $-1 \cdot 85$ & 0.06 & & \\
\hline & & Dhaka & $-2 \cdot 00$ & -0.04 & & & $-1 \cdot 81$ & 0.02 & & \\
\hline & & Khulna & $-1 \cdot 72$ & -0.32 & & & $-1 \cdot 61$ & $-0 \cdot 18$ & & \\
\hline & & Rajshahi & $-1 \cdot 88$ & $-0 \cdot 17$ & & & $-1 \cdot 61$ & $-0 \cdot 17$ & & \\
\hline & & Sylhet & $-2 \cdot 04$ & 0 & & & -1.79 & 0 & & \\
\hline & Mother's education level & No education & $-2 \cdot 17$ & $1 \cdot 20$ & $106 \cdot 40$ & $<0.001$ & -1.92 & 0.52 & $10 \cdot 72$ & $<0.001$ \\
\hline & & Primary & $-2 \cdot 09$ & $1 \cdot 13$ & & & -1.95 & 0.55 & & \\
\hline & & Secondary & $-1 \cdot 75$ & $0 \cdot 78$ & & & $-1 \cdot 82$ & $0 \cdot 42$ & & \\
\hline & & Higher & -0.97 & 0 & & & $-1 \cdot 40$ & 0 & & \\
\hline & Possession score & 0 & $-2 \cdot 22$ & $1 \cdot 06$ & $87 \cdot 29$ & $<0.001$ & -1.99 & 0.55 & $14 \cdot 05$ & $<0.001$ \\
\hline & & 1 & $-2 \cdot 10$ & 0.94 & & & $-1 \cdot 96$ & 0.52 & & \\
\hline & & 2 & $-1 \cdot 80$ & 0.65 & & & $-1 \cdot 79$ & $0 \cdot 35$ & & \\
\hline & & 3 & -1.59 & 0.44 & & & $-1 \cdot 68$ & $0 \cdot 24$ & & \\
\hline & & $4+$ & $-1 \cdot 16$ & 0 & & & $-1 \cdot 44$ & 0 & & \\
\hline & House type & All thatched & $-2 \cdot 28$ & 0.96 & $62 \cdot 30$ & $<0.001$ & $-1 \cdot 91$ & $0 \cdot 32$ & $4 \cdot 66$ & 0.001 \\
\hline & & Tin roof & $-2 \cdot 13$ & $0 \cdot 81$ & & & $-1 \cdot 87$ & $0 \cdot 27$ & & \\
\hline & & Tin wall and roof & $-2 \cdot 05$ & $0 \cdot 72$ & & & $-1 \cdot 79$ & $0 \cdot 20$ & & \\
\hline & & Brick wall/roof/floor & $-1 \cdot 68$ & $0 \cdot 36$ & & & $-1 \cdot 69$ & 0.09 & & \\
\hline & & All brick & $-1 \cdot 32$ & 0 & & & $-1 \cdot 59$ & 0 & & \\
\hline \multirow[t]{6}{*}{ WHZ } & Region & Barisal & $-0 \cdot 74$ & -0.23 & $7 \cdot 59$ & $<0.001$ & -0.67 & -0.21 & $4 \cdot 56$ & $<0.001$ \\
\hline & & Chittagong & -0.97 & -0.01 & & & -0.89 & 0.02 & & \\
\hline & & Dhaka & -0.90 & -0.07 & & & -0.83 & -0.05 & & \\
\hline & & Khulna & -0.97 & 0.00 & & & $-0 \cdot 87$ & -0.01 & & \\
\hline & & Rajshahi & -1.07 & $0 \cdot 10$ & & & -0.94 & 0.06 & & \\
\hline & & Sylhet & -0.97 & 0 & & & $-0 \cdot 88$ & 0 & & \\
\hline
\end{tabular}

WAZ, weight-for-age Z-score; HAZ, height-for-age Z-score; WHZ, weight-for-height Z-score.

very few $(2 \cdot 1 \%)$ were wasted only. Nearly one-fifth $(19 \cdot 6 \%)$ of the children were severely stunted. Underweight and wasting prevalences were $40.7 \%$ and $14.3 \%$, respectively, of whom $12.5 \%$ were severely underweight and $3.2 \%$ were severely wasted.

Only those variables having a significant association with nutritional status of the children are presented in the tables. No significant difference between mean WAZ, HAZ and WHZ of male and female children was found, both before and after removing age effects. Table 2 shows that after correction for the other demographic and socio-economic variables, in analysis 2 , mother's educational level and number of possessions showed strong positive associations with WAZ and HAZ; as the mother's educational level and number of possessions increased so the child's mean WAZ and HAZ improved. The difference in mean WAZ and HAZ between owning $4+$ possessions and none was over half a standard deviation and for no education to higher education of mothers was $0.4 \mathrm{SD}$.
Children living in households with a sanitary toilet had, on average, a better WAZ than those living in households with either an unsanitary latrine or no toilet. Upward trends of HAZ means were evident from poor to good housing. Regional heterogeneity in HAZ was also observed, with poorer HAZ mean in Barisal, Chittagong and Sylhet districts. Regional variation was also found for WHZ; children from Rajshahi district had the worst mean value $(-1 \cdot 07)$, whereas those from Barisal district had the best mean $(-0 \cdot 74)$. If a sequential Bonferroni correction was applied then the number of possessions would be just significant, with an upward trend in mean from no to $4+$ possessions. Binary logistic regression analyses were undertaken to see how well the socio-economic and demographic variables predicted underweight, stunting and wasting (Table 3) and no significant relationships were found with WHZ. When all of the variables were entered together, the overall models (analysis 2) explained 18.5\% of the variation in HAZ, $10.9 \%$ of WAZ and $3.0 \%$ of WHZ. 
Table 3 Binary logistic regression analysis results of Z-scores v. socio-economic and demographic variables: children aged $<5$ years, Bangladesh Demographic and Health Survey 2004

\begin{tabular}{|c|c|c|c|c|c|c|c|c|c|c|c|c|c|c|}
\hline & & & \multicolumn{6}{|c|}{ Analysis 1} & \multicolumn{6}{|c|}{ Analysis 2} \\
\hline & & & \multicolumn{2}{|c|}{$\%$ predicted $^{\star}$} & \multirow[b]{2}{*}{$\chi^{2}$} & \multirow[b]{2}{*}{$P$} & \multirow[b]{2}{*}{ OR } & \multirow[b]{2}{*}{$95 \% \mathrm{Cl}$} & \multicolumn{2}{|c|}{$\%$ predicted $^{\star}$} & \multirow[b]{2}{*}{$x^{2}$} & \multirow[b]{2}{*}{$P$} & \multirow[b]{2}{*}{ OR } & \multirow[b]{2}{*}{$95 \% \mathrm{Cl}$} \\
\hline & & & 1 & 2 & & & & & 1 & 2 & & & & \\
\hline \multirow[t]{9}{*}{ WAZ } & Mother's education level & No education & $81 \cdot 0$ & $33 \cdot 1$ & $168 \cdot 82$ & $<0.001$ & $5 \cdot 31$ & $3 \cdot 80,7 \cdot 43$ & $78 \cdot 9$ & $38 \cdot 5$ & $16 \cdot 75$ & 0.001 & $2 \cdot 28$ & $1 \cdot 47,3.51$ \\
\hline & & Primary & & & & & $4 \cdot 14$ & $2 \cdot 95,5 \cdot 80$ & & & & & $2 \cdot 05$ & $1 \cdot 34,3 \cdot 14$ \\
\hline & & $\begin{array}{l}\text { Secondary } \\
\text { Highert }\end{array}$ & & & & & $2 \cdot 69$ & $1 \cdot 91,3 \cdot 79$ & & & & & $1 \cdot 75$ & $1 \cdot 16,2 \cdot 62$ \\
\hline & Possession score & 0 & $78 \cdot 8$ & $35 \cdot 7$ & $189 \cdot 47$ & $<0.001$ & $4 \cdot 34$ & $3 \cdot 29,5 \cdot 73$ & $78 \cdot 9$ & $38 \cdot 5$ & $38 \cdot 32$ & $<0.001$ & $2 \cdot 44$ & $1 \cdot 75,3 \cdot 41$ \\
\hline & & 1 & & & & & $3 \cdot 63$ & $2 \cdot 72,4 \cdot 82$ & & & & & $2 \cdot 34$ & $1 \cdot 70,3 \cdot 24$ \\
\hline & & 2 & & & & & $2 \cdot 48$ & $1 \cdot 84,3 \cdot 33$ & & & & & $1 \cdot 86$ & $1 \cdot 35,2 \cdot 56$ \\
\hline & & $\begin{array}{l}3 \\
4+t\end{array}$ & & & & & $1 \cdot 83$ & $1 \cdot 33,2 \cdot 52$ & & & & & $1 \cdot 50$ & $1 \cdot 07,2 \cdot 10$ \\
\hline & Toilet & No toilet & $84 \cdot 6$ & $26 \cdot 4$ & $120 \cdot 48$ & $<0.001$ & $2 \cdot 83$ & $2 \cdot 30,3 \cdot 48$ & $78 \cdot 9$ & $38 \cdot 5$ & $14 \cdot 21$ & 0.001 & $1 \cdot 61$ & $1 \cdot 26,2 \cdot 07$ \\
\hline & & $\begin{array}{l}\text { Insanitary } \\
\text { Sanitaryt }\end{array}$ & & & & & $1 \cdot 88$ & $1 \cdot 63,2 \cdot 17$ & & & & & $1 \cdot 21$ & $1 \cdot 02,1 \cdot 44$ \\
\hline \multirow[t]{16}{*}{$\mathrm{HAZ}$} & Region & Barisal & $57 \cdot 1$ & $64 \cdot 6$ & $39 \cdot 71$ & $<0.001$ & $1 \cdot 19$ & $0.93,1.51$ & $62 \cdot 0$ & $70 \cdot 6$ & $19 \cdot 92$ & 0.001 & $1 \cdot 20$ & $0.92,1.56$ \\
\hline & & Chittagong & & & & & 0.99 & $0 \cdot 80,1 \cdot 22$ & & & & & 1.01 & $0 \cdot 80,1 \cdot 26$ \\
\hline & & Dhaka & & & & & 0.93 & $0 \cdot 76,1 \cdot 14$ & & & & & 0.97 & $0 \cdot 77,1 \cdot 23$ \\
\hline & & Khulna & & & & & $0 \cdot 62$ & $0.49,0.77$ & & & & & 0.75 & $0.58,0.97$ \\
\hline & & $\begin{array}{l}\text { Rajshahi } \\
\text { Svlhett }\end{array}$ & & & & & 0.79 & $0.64,0.98$ & & & & & $0 \cdot 76$ & $0.60,0.97$ \\
\hline & Mother's education level & No education & $57 \cdot 8$ & $67 \cdot 4$ & $200 \cdot 23$ & $<0.001$ & $6 \cdot 77$ & $4 \cdot 87,9 \cdot 41$ & $62 \cdot 0$ & $70 \cdot 6$ & $16 \cdot 91$ & 0.001 & $2 \cdot 36$ & $1 \cdot 54,3 \cdot 61$ \\
\hline & & Primary & & & & & $5 \cdot 71$ & $4 \cdot 10,7 \cdot 95$ & & & & & $2 \cdot 30$ & $1.52,3.50$ \\
\hline & & $\begin{array}{l}\text { Secondary } \\
\text { Highert }\end{array}$ & & & & & $3 \cdot 73$ & $2 \cdot 67,5 \cdot 21$ & & & & & $2 \cdot 11$ & $1 \cdot 42,3 \cdot 14$ \\
\hline & Possession score & 0 & $59 \cdot 7$ & $68 \cdot 3$ & $236 \cdot 91$ & $<0.001$ & $5 \cdot 14$ & $3 \cdot 93,6 \cdot 71$ & $62 \cdot 0$ & $70 \cdot 6$ & $44 \cdot 61$ & $<0.001$ & $2 \cdot 52$ & $1 \cdot 82,3 \cdot 49$ \\
\hline & & 1 & & & & & 4.55 & $3 \cdot 46,6 \cdot 00$ & & & & & $2 \cdot 54$ & $1 \cdot 85,3 \cdot 47$ \\
\hline & & 2 & & & & & $2 \cdot 86$ & $2 \cdot 15,3 \cdot 81$ & & & & & $1 \cdot 86$ & $1 \cdot 37,2 \cdot 54$ \\
\hline & & $\begin{array}{l}3 \\
4++\end{array}$ & & & & & $2 \cdot 10$ & $1 \cdot 55,2 \cdot 85$ & & & & & $1 \cdot 56$ & $1 \cdot 13,2 \cdot 16$ \\
\hline & House type & All thatched & $59 \cdot 4$ & $67 \cdot 8$ & $172 \cdot 24$ & $<0.001$ & $4 \cdot 51$ & $3 \cdot 39,6 \cdot 01$ & $62 \cdot 0$ & $70 \cdot 6$ & $16 \cdot 18$ & 0.003 & 1.90 & $1 \cdot 35,2 \cdot 67$ \\
\hline & & Tin roof & & & & & $3 \cdot 52$ & $2 \cdot 79,4 \cdot 44$ & & & & & $1 \cdot 73$ & $1 \cdot 29,2 \cdot 30$ \\
\hline & & Tin wall and roof & & & & & $3 \cdot 30$ & $2 \cdot 60,4 \cdot 17$ & & & & & 1.66 & $1 \cdot 24,2 \cdot 23$ \\
\hline & & $\begin{array}{l}\text { Brick wall/roof/floor } \\
\text { All brickt }\end{array}$ & & & & & $2 \cdot 06$ & $1 \cdot 59,2 \cdot 68$ & & & & & 1.45 & $1 \cdot 09,1.93$ \\
\hline
\end{tabular}

WAZ, weight-for-age Z-score; HAZ, height-for-age Z-score.

Note: no significant result was found for weight-for-height $Z$-score.

${ }^{*} 1=$ normal; 2 = malnourished.

tReference group. 
After adjustment for the other socio-economic and demographic variables, children of less educated mothers and from households having fewer possessions were more likely to be underweight, as well as stunted. For example, children with no educated mothers were $2 \cdot 28$ and 2.36 times more likely to be underweight and stunted, respectively, than children whose mothers had higher education. Children having no toilet facilities in their house were 1.61 times more likely to be underweight compared with children having sanitary latrine facilities; whereas children from all-thatched households were 1.90 times more likely to be stunted compared with children from all-brick households. No significant associations were found between wasting and any of the socio-economic and demographic variables (results not shown). Prediction of normal children by the models varied from $62 \%$ to $100 \%$, while $39 \%$ of those underweight, $71 \%$ of those stunted and only $0.1 \%$ of the wasted children were correctly predicted.

A comparison of the percentages of stunting, underweight and wasting using the quintiles from the poverty index and the categories of possession score is presented in Table 4 . The percentage of stunted children varied from $26 \cdot 3 \%$ to $13 \cdot 7 \%$ in the poorest and richest quintiles, respectively, while the range was $45 \cdot 6 \%$ to $3 \cdot 4 \%$ for possession score of 0 and $4+$. For underweight children, corresponding percentages ranged from $28.1 \%$ to $13.0 \%$ for the poverty index and from $46.7 \%$ to $3.5 \%$ for possession score. The percentage difference of wasted children in the richest and poorest quintile was $12.3 \%$ only, whereas it was $39 \cdot 2 \%$ between owners of 0 and $4+$ possessions. Table 4 also shows that the average difference between possession score categories was much larger than between poverty index quintiles, e.g. stunting $10.5 \%$ and $3.3 \%$, respectively. Regression analyses showed that the slopes of the lines were all significantly greater for possession score than for poverty index.

Overall, these results suggest that the possession score provided greater discrimination than the poverty index (minimum $39 \%$ in possession score (wasting) $v$. maximum $15 \%$ (underweight) in poverty index). GLM analyses (tables not shown) found that the mean $Z$-score differences between the richest and poorest generated by possession score were greater compared with poverty index. Binary logistic regression analyses were also used to estimate the predictive accuracy of poverty index in identifying underweight or stunted children, which revealed that the correct identification rate of underweight children was $4.5 \%$ lower when poverty index rather than possession score was used. Both of these indicate better discriminatory power of the possession score over the poverty index.

Table 5 presents the various combinations of stunting, wasting and underweight in relation to poverty index and possession score. The possession score provides much greater discrimination of the children who were stunted,

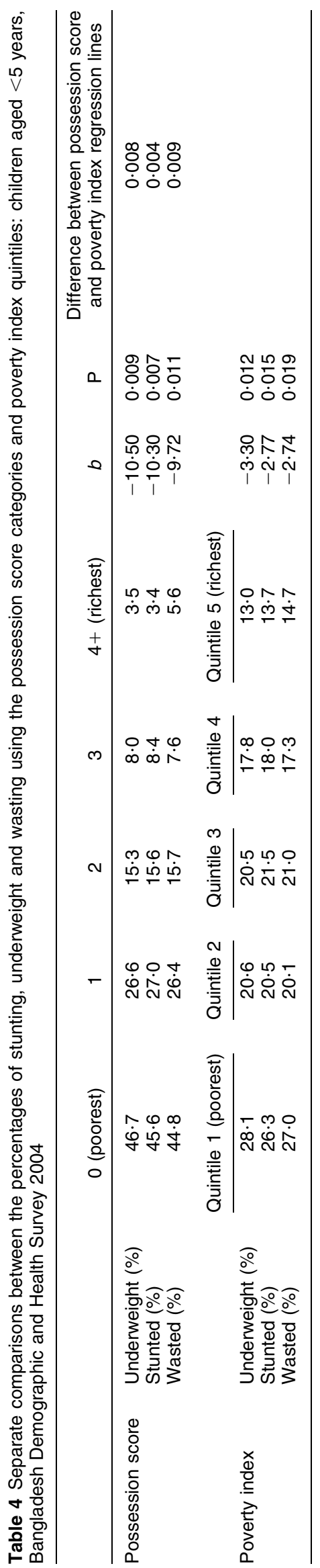


wasted and underweight: $49 \cdot 1 \%$ lived in houses with no possessions compared with only $4 \cdot 2 \%$ of children with $4+$ possessions. The equivalent percentages for the poverty index were $28.6 \%$ and $11 \cdot 4 \%$. With the possession score all of the percentages decreased from 0 to $4+$ possessions, whereas this was not found using the poverty index. Furthermore, only $58.4 \%$ of children in the richest quintile were not stunted, wasted or underweight compared with $71 \cdot 0 \%$ of children living in households with $4+$ possessions.

\section{Discussion and conclusions}

Chronic undernutrition was very common in these Bangladeshi children aged $<5$ years: more than half of the children were suffering from stunting or stunting with underweight or wasting, while very few children were observed with only acute undernutrition. This result implies a long-term burden of frequent infections and poor diet.

Numerous studies have documented gender differences in health and nutritional status in developing countries, generally finding that boys do better than girls $^{(11)}$. In patriarchal societies it is common for the intrahousehold allocation of resources to strongly favour sons, to the detriment of daughters' health and educational status. The present study, however, found that there was no obvious, overall gender differential in child nutritional status; underweight, stunting and wasting prevalences were almost identical in boys and girls. Bangladesh is generally thought to be a patriarchal society, but the apparent lack of a gender differential in undernutrition indicates that intra-household gender bias in feeding and health care for children in Bangladesh may be much more equal than hitherto suspected. Paternal education was not found to be associated with any of the malnutrition indicators after adjusting for the other socio-economic and demographic variables and with Bonferroni correction; whereas, contradicting many other studies ${ }^{(12)}$, maternal education showed strong associations with childhood underweight and stunting, even after controlling for all socio-economic and demographic variables and with Bonferroni correction. Thus female education should still be one of the key policy options to achieve the MDG on malnutrition in Bangladesh. After controlling for all socio-economic and demographic factors, a trend of improvement in mean WAZ and HAZ was evident with possession score, indicating a strong association of malnutrition and socio-economic factors.

Moreover, the current study suggests that the simple and easy-to-construct possession score is a better indicator of SES, as this score gives greater discrimination in identifying malnourished children than a poverty index. The poorer discrimination using the poverty index may be because there was insufficient variation in assets; 
it was evident that the first principal component generated from the Bangladesh Demographic and Health Survey data described only $31.7 \%$ of the variation of the assets. This limitation of the PCA technique was also observed in the Baseline Result and Impact Management System Survey conducted in Bangladesh in 2006(13), where the distribution of different assets used for constructing the poverty index showed little variation among households; for example, the floor material was essentially the same and very few $(0 \cdot 3 \%)$ owned a car.

Urban-rural discrepancies in malnutrition seem to be reducing, thus current policies need to address urban malnutrition. Education (especially of females) should still be one of the key policy options to achieve the MDG on malnutrition in Bangladesh. The multifaceted nature of malnutrition means that it may be effectively addressed only when several sectors and strategies work together. So, for example, combining income-generation projects and infant feeding programmes together with better household access to food (food security), more accessible health services, regular deworming of children and improved sanitation, can bring the desirable nutritional status.

In conclusion, the current study has shown that undernutrition in Bangladeshi children aged $<5$ years has strong associations with poor SES, which can be measured by a simple asset count or possession score. Maternal education is a key variable in improving nutritional status among young children.

\section{Acknowledgements}

Source of funding: The study was supported by grants from the Cambridge Commonwealth Trust. Conflict of interest declaration: None of the authors has any conflicts of interest to declare. Authorship responsibilities: M.M. reviewed the literature, prepared the data, performed the statistical analysis and wrote the first draft. C.G.N.M.-T. contributed to study design, data analysis, interpretation and manuscript preparation. R.G. contributed conceptual and statistical advice.

\section{References}

1. Black RE, Allen LH, Bhutta ZA et al. (2008) Maternal and child undernutrition: global and regional exposures and health consequences. Lancet 371, 243-260.

2. Ezzati M, Lopez AD, Rodgers A et al. (2002) Selected major risk factors and global and regional burden of disease. Lancet 360, 1347-1360.

3. United Nations Standing Committee on Nutrition (2000) Nutrition Throughout the Life Cycle, 4th Report on the World Nutrition Situation. Geneva: SCN.

4. United Nations (2005) The Millennium Development Goals Report 2005. New York: UN.

5. Fotso J-C \& Kuate-Defo B (2005) Measuring socioeconomic status in health research in developing countries: should we be focusing on households, communities or both? Soc Indic Res 72, 189-237.

6. Pollack CE, Chideya S, Cubbin C et al. (2007) Should health studies measure wealth? A systematic review. Am J Prev Med 33, 250-264.

7. Helen Keller International \& Institute of Public Health Nutrition (2006) Housebold and Community Level Determinants of Malnutrition in Bangladesh. Dhaka: HKI/ IPHN.

8. Semba RD, de Pee S, Sun K et al. (2008) Effect of parental formal education on risk of child stunting in Indonesia and Bangladesh: a cross-sectional study. Lancet 371, 322-328.

9. Zere E \& McIntyre D (2003) Inequities in under-five child malnutrition in South Africa. Int J Equity Health 2, 7.

10. Houweling TA, Kunst AE \& Mackenbach JP (2003) Measuring health inequality among children in developing countries: does the choice of the indicator of economic status matter? Int J Equity Health 2, 8.

11. Schoenbaum M, Tulchinsky TH \& Abed Y (1995) Gender differences in nutritional status and feeding patterns among infants in the Gaza Strip. Am J Public Health 85, 965-969.

12. Desai S \& Alva S (1998) Maternal education and child health: is there a strong causal relationship? Demography 35, 71-81.

13. International Fund for Agricultural Development (2005) Pilot Baseline Survey. Dhaka: TANGO International and IFAD. 